\title{
Aislamiento de esporas y evaluación de métodos de inoculación en la producción de micorrizas en cultivos trampa
}

Spore isolation and evaluation of inoculation methods in the production of mycorrhizae in trap crops

Wendy Aguilar-Ulloa ${ }^{1}$, Priscilla Arce-Acuña ${ }^{2}$, Fiorella Galiano-Murillo, Terry J. Torres-Cruz ${ }^{4}$

Fecha de recepción: 27 de marzo del 2015

Fecha de aprobación: 6 de agosto del 2015

Aguilar-Ulloa, W; Arce-Acuña, P; Galiano-Murillo, F; TorresCruz, T. Aislamiento de esporas y evaluación de métodos de inoculación en la producción de micorrizas en cultivos trampa. Tecnología en Marcha. Edición Especial Biocontrol.

Pág 5-14.

Estudiante de Ingeniería en Biotecnología, Instituto Tecnológico de Costa Rica. Tel. 86879975, correo electrónico: w.aguilar0@hotmail.com

2 Estudiante de Ingeniería en Biotecnología, Instituto Tecnológico de Costa Rica. Tel. 6052-0515, correo electrónico:priarce.30@gmail.com

3 Estudiante de Ingeniería en Biotecnología, Instituto Tecnológico de Costa Rica. Tel. 88768755, correo electrónico: fiogaliano@hotmail.com

4 Estudiante de Ingeniería en Biotecnología, Instituto Tecnológico de Costa Rica. Tel. 84401809, correo electrónico: terryjari@gmail.com 


\title{
Palabras clave
}

Micorriza; simbiosis; esporas; Phaseolus vulgaris; crecimiento.

\section{Resumen}

Los microorganismos del suelo desempeñan un importante papel en la agricultura; entre ellos están las micorrizas que generan una gran cantidad de beneficios para las plantas. En la presente investigación se tomaron muestras de suelo y raíz de plantas de vainica; se aislaron esporas por el método de tamizado y decantación; se realizó conteo de esporas y tinción de raíces y luego se determinó el porcentaje de colonización. Además, se inocularon 15 macetas con cada uno de los diferentes tratamientos (esporas, suelo y raíces) y se sembraron semillas de frijol en cada una. Luego de un mes, se midió la longitud de la raíz y el tallo de todos los tratamientos. Además, para determinar el desarrollo del inóculo en cada tratamiento se precisó la cantidad de esporas producidas y el nivel de colonización de las raíces. La longitud del tallo y de la raíz de las plantas evaluadas no presentó diferencias estadísticamente significativas entre los distintos tratamientos. El método de inoculación con esporas presentó el mayor porcentaje de micorrización. Los métodos de inoculación que favorecieron más la producción de estructuras reproductivas fueron los tratados con las esporas aisladas y los tratados con raíces; de igual forma, ambos presentaron la mayor cantidad de esporas germinadas. Para cultivos como frijol o vainica, se recomienda utilizar como inóculo las raíces de plantas infectadas.

\section{Keywords}

Mycorrhiza; symbiosis; Phaseolus vulgaris.

\begin{abstract}
Soil microorganisms play an important role in agriculture, among them we find mycorrhizae, which generate to the plant a great quantity of benefits. In this investigation we took soil and root samples from bean plants. Spores were isolated by the method of wet sieving and decanting. We did a spore count and stained roots and then determined the percentage of AMF colonization. We inoculated 15 pots with each treatment; spores, soil and roots, and we planted bean seeds on each one of them. A month later we measured the length of the roots and stems of every treatment. Also, to determine the inoculum development we determined the quantity of spores produced and the percentage of AMF colonization of the roots. Roots and stems length of the plants evaluated did not present statistically significant differences among the different treatments. The spore inoculation method presented the higher mycorrhization percentage. The methods of inoculation that favored the production of reproductive structures were the treatments with isolated spores and roots; also both of them presented the higher quantity of germinated spores. We recommend for use in crops like beans to use root inoculum from infected plants.
\end{abstract}

\section{Introducción}

En la actualidad, la necesidad de obtener cultivos con altos rendimientos y calidad en periodos cortos de tiempo ha llevado al empleo de prácticas agronómicas que dependen de productos agroquímicos. Sin embargo, estas prácticas pueden causar un impacto negativo sobre el medio y con ello la degradación de los recursos naturales, la erosión genética y la contaminación ambiental (Pérez, Rojas \& Montes, 2011; Terry et al., 2013). Ante esta situación, la biotecnología agrícola se ha convertido en un campo importante de conocimiento científico y de nuevas 
tecnologías que tienen como finalidad principal reducir el uso de productos químicos peligrosos y prácticas agrícolas que tengan efectos perjudiciales sobre el entorno, a la vez que se mantienen o aumentan los rendimientos (Terry et al., 2013).

Los microorganismos del suelo desempeñan un papel importante en el contexto agrícola, debido a que contribuyen al funcionamiento de los ecosistemas terrestres, ya que permiten tanto la recuperación de suelos dañados como la sustitución parcial o total de los fertilizantes minerales; además de su bajo costo de producción y la posibilidad de ser producidos a partir de recursos renovables (Cruz et al., 2014). Para estudiar las comunidades de organismos y ecosistemas se han desarrollado diversas investigaciones. Por ejemplo, los hongos son los organismos más estudiados debido a su papel como descomponedores primarios y su participación en los ciclos biogeoquímicos (Cuadros et al., 2011).

Los hongos formadores de micorrizas son uno de los componentes principales de las comunidades microbianas rizosféricas que permiten establecer relaciones de simbiosis con alrededor del $90 \%$ de las plantas vasculares. Son importantes principalmente para lograr una mayor absorción de nutrientes, niveles mayores en la producción de hormonas y clorofila, incremento en la vida útil de las raíces, tolerancia al estrés (abiótico y biótico), mejora de las condiciones del suelo y en el establecimiento de relaciones sinérgicas con otros microorganismos (Paillacho, 2010; Mohammadi et al., 2011). Por lo tanto, ha cobrado gran importancia el estudio de técnicas para aislar y evaluar el rendimiento de estos organismos con el fin de aplicarlos al suelo como biofertilizantes, ya que constituyen una alternativa para la solución de problemas de propagación, aclimatación y nutrición, al reducir los costos de producción y permitir sistemas más eficientes y sostenibles (CORPOICA, 2008; Cruz et al., 2014).

El frijol o vainica (Phaseolus vulgaris L.) es la leguminosa más importante en el consumo humano a nivel mundial, especialmente en Centroamérica y Suramérica, debido a sus cualidades nutritivas, diversidad de variedades y alto contenido de proteína (Ulloa et al., 2011). Este cultivo ha sido utilizado en varias investigaciones como trampa para la producción de micorrizas, por su susceptibilidad para ser colonizado por ellas y su rápido crecimiento (Paillacho, 2010; Liriano et al., 2012). En esta investigación se evalúan varios métodos de inoculación para producir micorrizas en cultivos trampa de vainica.

\section{Metodología}

Las muestras se obtuvieron en un cultivo de vainicas de la zona de Carrillos Altos de Poás, provincia de Alajuela. Para tomar muestras de la rizosfera de la planta se utilizó un palín, haciendo un corte de $15 \mathrm{~cm}$ de profundidad, $20 \mathrm{~cm}$ de ancho y $20 \mathrm{~cm}$ de largo. Las muestras se colocaron en bolsas y se desmenuzaron. También se tomaron muestras de raíces, que se mantuvieron en refrigeración hasta el momento de los análisis.

\section{Tinción de raíces}

Se pesó $1 \mathrm{~g}$ de raíces (secundarias y terciarias) y se lavaron por dos minutos con agua abundante, luego se colocaron en tubos Falcon. Se procedió a realizar la clarificación adicionando hidróxido de potasio $(\mathrm{KOH})$ al 2,5\% hasta que todas las raíces quedaron cubiertas y se mantuvieron en baño maría a $90{ }^{\circ} \mathrm{C}$ durante una hora. Posteriormente, se decantó el hidróxido de potasio $(\mathrm{KOH})$ y se adicionó ácido clorhídrico $(\mathrm{HCl})$ al $2 \%$ durante una hora a temperatura ambiente. Luego se decantó el $\mathrm{HCl}$ y se lavaron las raíces con agua. Finalmente, se agregó tinta china como colorante y se dejó en baño maría a $90^{\circ} \mathrm{C}$ durante una hora; transcurrido este tiempo se decantó y las raíces se depositaron en placas de Petri con glicerol. Las placas se mantuvieron refrigeradas hasta el día de la evaluación. 


\section{Determinación del nivel de colonización}

Por cada unidad experimental se prepararon cuatro placas y en cada una se colocaron cinco trozos de raíces teñidas. Las raíces se observaron utilizando un microscopio de luz en objetivo 10X para contar los campos colonizados con estructuras de micorrizas dentro de ellas (hifas, arbúsculos y vesículas) y los no colonizados. En algunos casos se requirió utilizar el objetivo 40X para detallar las diferentes estructuras de las micorrizas. El porcentaje de colonización se determinó mediante la observación de las placas en el microscopio dividiendo el número de campos colonizados por el total de campos observados y multiplicando el resultado por 100.

$$
\% \text { colonización }=\left(\frac{\text { campos colonizados }}{\text { campos totales observados }}\right) \times 100
$$

\section{Aislamiento de esporas}

Se tomaron cuatro muestras de suelo de $25 \mathrm{~g}$ y se colocaron en beakers. Los agregados de suelo se trituraron con una espátula. A cada beaker se le añadieron $250 \mathrm{~mL}$ de agua destilada y las suspensiones se agitaron por una hora. Estas suspensiones se hicieron pasar por tamices apilados (850 $\mu \mathrm{m}, 250 \mu \mathrm{m}, 106 \mu \mathrm{m}$ y $63 \mu \mathrm{m})$. Se añadió agua del tubo para facilitar el movimiento de las esporas. El material retenido en los tamices de $106 \mu \mathrm{m}$ y $63 \mu \mathrm{m}$ se colocó en tubos Falcon y se suspendió en agua, se centrifugó por tres minutos a 2000 g y se removió el sobrenadante. El sedimento se resuspendió en una solución de sacarosa al 50\% y se centrifugó por dos minutos a $2000 \mathrm{~g}$. El sobrenadante se hizo pasar por un papel filtro Whatman 5 y se hicieron dos lavados con agua destilada, utilizando un sistema de filtración al vacío. Los diferentes papeles filtros se colocaron en placas de Petri y se mantuvieron en refrigeración hasta el momento del conteo.

\section{Conteo de esporas}

Los papeles filtro en los cuales se retuvieron las esporas, colocados en placas de Petri, se observaron con un estereoscopio para realizar el conteo del número de esporas presentes y del número de esporas germinadas presentes en las muestras.

\section{Pruebas de inoculación para cultivos trampa}

Para determinar el efecto del método de inoculación en la producción de cultivos trampa se realizaron pruebas con plantas de vainica utilizando diferentes tratamientos: testigo, inoculación con esporas, inoculación con suelo e inoculación con raíces. Para realizar la inoculación con esporas, se utilizaron las obtenidas con el método de tamizaje y decantación, previamente descrito. Se seleccionaron las esporas utilizando el microscopio y se colocaron en papel filtro en triángulos. Para iniciar el cultivo se utilizaron aproximadamente 200 esporas por maceta. Las esporas en los triángulos de filtro se lavaron en un orificio de $2 \mathrm{~cm}$ de profundidad realizado en el sustrato de cada una de las macetas (figura 1a). Se cubrieron con tierra y se incubaron por cuatro días, antes de sembrar sobre ellas las semillas de la planta huésped. Para la inoculación con suelo se pesaron $100 \mathrm{~g}$ de la muestra de suelo seco y se mezclaron en igual proporción con tierra estéril. Para el montaje del tratamiento con inoculación con suelo en las macetas se agregó una capa de tierra estéril, seguida de la mezcla de tierra con la muestra de suelo y finalmente otra capa de tierra estéril (figura 1b). Posteriormente, se sembraron las semillas de la planta hospedera. En el caso de la inoculación con raíces, estas se colocaron en una maceta con tierra estéril como se muestra en la figura 1c. Luego se sembraron las semillas de la planta hospedera. Las macetas se mantuvieron en invernadero durante el tiempo de la prueba. 


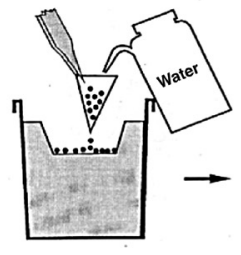

a

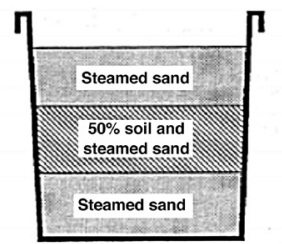

b

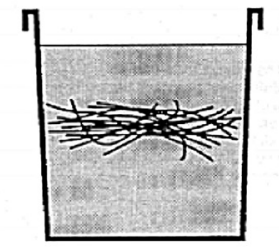

C

Figura 1. Inoculación con micorrizas. a) inoculación con esporas, b) inoculación con suelo y c) inoculación con raíces. Fuente: Habte y Osorio, 2001.

\section{Evaluación del inóculo producido en los cultivos trampa}

Transcurrido un mes, se midió la longitud de la raíz y del tallo de todas las muestras. Para determinar el desarrollo del inóculo se obtuvo la cantidad de esporas producidas mediante el método de tamizaje y decantación y el nivel de colonización de las raíces. En este caso, para el nivel de colonización se realizaron las siguientes modificaciones al procedimiento anteriormente descrito: se utilizó $\mathrm{KOH}$ al $10 \%$ durante 15 minutos, $\mathrm{HCl}$ al 10\% durante 12 minutos y la tinción se agregó al fondo de los tubos durante 15 minutos. Además, en lugar de glicerol se utilizó una gota de aceite de inmersión para fijar las muestras al portaobjetos.

\section{Resultados}

Luego de verificar la normalidad o no de los datos se realizó la prueba de Kruskal-Wallis, con la comparación de pares para los resultados de la longitud de tallo y raíz de las plantas evaluadas en los diferentes tratamientos, y se encontró que no existía una diferencia estadísticamente significativa entre los tratamientos $\left(p_{\text {raiz }}=0,7442 ; p_{\text {tallo }}=0,1957\right)$ tanto para raíz como para tallo, con un $5 \%$ de significancia. Se realizó un ANOVA para el número de esporas totales y las esporas germinadas de cada uno de los tratamientos con una significancia del 5\%; se encontró que estadísticamente existía una diferencia significativa entre los tratamientos con respecto al número de esporas totales $(p=0,0049)$, mientras que no había diferencia significativa entre los tratamientos con respecto al número de esporas germinadas $(p=0,0782)$. En el cuadro 1 se presentan las medias del número de esporas totales y el número de esporas germinadas obtenidas con la prueba de Tuckey, en la cual la media mayor es la del tratamiento de las esporas. Estadísticamente, los tratamientos con inóculo de esporas e inóculo de raíces presentan un mejor rendimiento en la germinación de esporas.

Cuadro 1. Prueba de Tuckey para el número de esporas y la germinación de las mismas, obtenida en los distintos tratamientos de inoculación.

\begin{tabular}{|c|c|c|}
\hline Tratamiento & $\begin{array}{c}\text { Media de número de } \\
\text { esporas totales }\end{array}$ & $\begin{array}{c}\text { Media de número de } \\
\text { esporas germinadas }\end{array}$ \\
\hline Testigo & $121,60 \mathrm{~A}$ & $1,00 \mathrm{~A}$ \\
\hline Inoculación con suelo & $170,60 \mathrm{AB}$ & $1,80 \mathrm{AB}$ \\
\hline Inoculación con esporas & $233,60 \mathrm{~B}$ & $3,80 \mathrm{~A}$ \\
\hline Inoculación con raíces & $232,40 \mathrm{~B}$ & $4,60 \mathrm{~A}$ \\
\hline
\end{tabular}


En el cuadro 2 se presenta el porcentaje de colonización inicial y de los diferentes tratamientos evaluados, observándose que el tratamiento de inoculación con esporas fue incluso mayor que el porcentaje inicial de colonización.

Cuadro 2. Porcentaje de colonización de las micorrizas mediante los diferentes tratamientos.

\begin{tabular}{|c|c|}
\hline Tratamiento & Porcentaje de colonización \\
\hline Inicial & $70 \%$ \\
\hline Testigo & $10 \%$ \\
\hline Inoculación con esporas & $75 \%$ \\
\hline Inoculación con suelo & $25 \%$ \\
\hline Inoculación con raíz & $25 \%$ \\
\hline
\end{tabular}

En la figura 2 se muestran las estructuras micorrícicas observadas en los tratamientos durante el conteo de esporas y en la determinación del porcentaje de colonización.

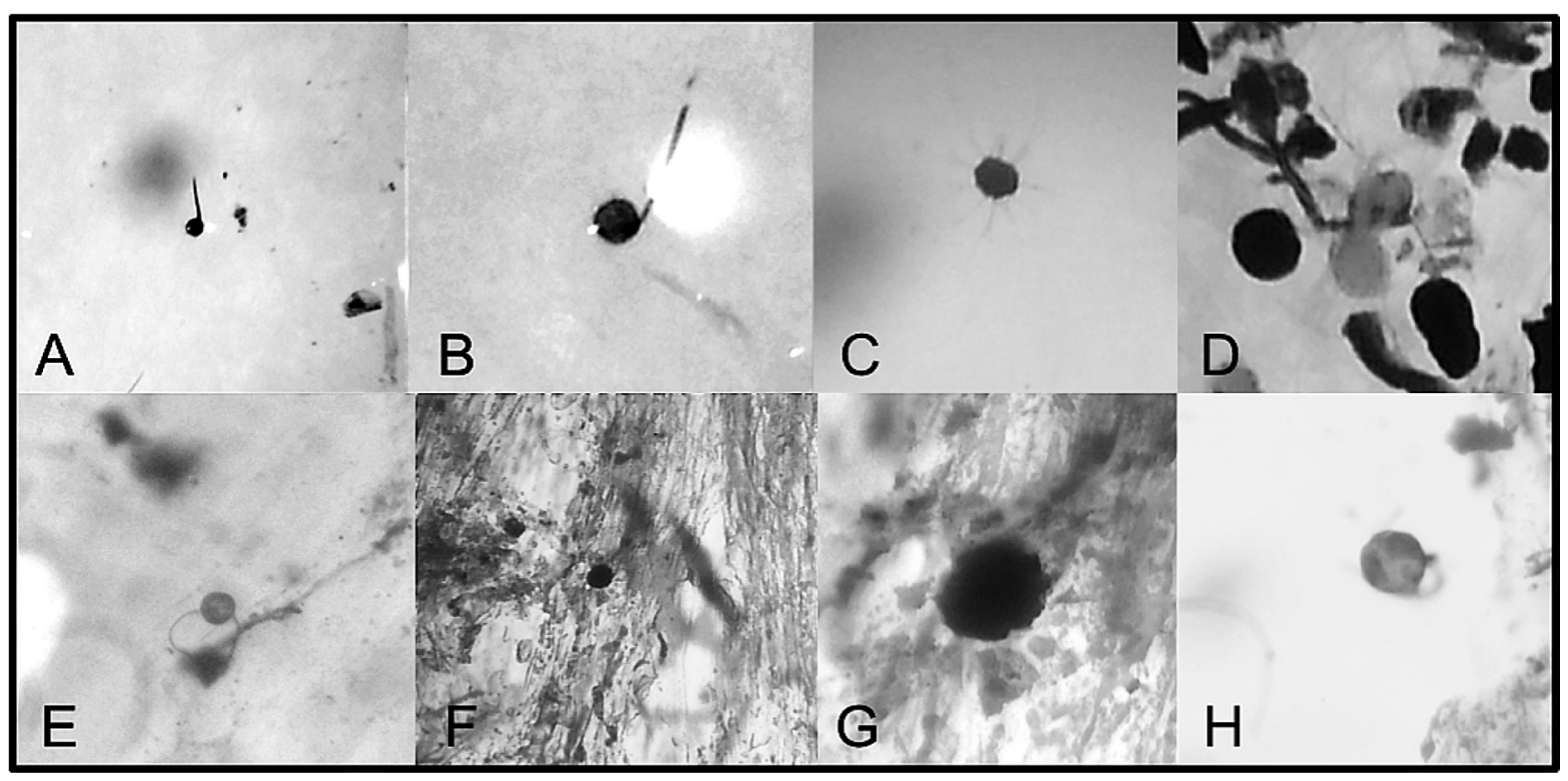

Figura 2. Esporas observadas en los distintos tratamientos. Durante el conteo de esporas: A) Germinada en el control (30X). B) Germinada en inoculación con esporas (30X). C) Germinada en inoculación con suelo (30X). D) Germinada (blanca transparente) en inoculación con raíz (30X). Durante la determinación del nivel de colonización: E) Espora observada en el control (1000X). F) Espora observada en inoculación con esporas (400X). G) Arbúsculo observado en inoculación con suelo (1000X). H) Espora observada en inoculación con raíz (1000X).

En la figura 3 se muestra la morfología de las esporas observadas en los tratamientos evaluados, caracterizadas por su coloración, tamaño y forma. 


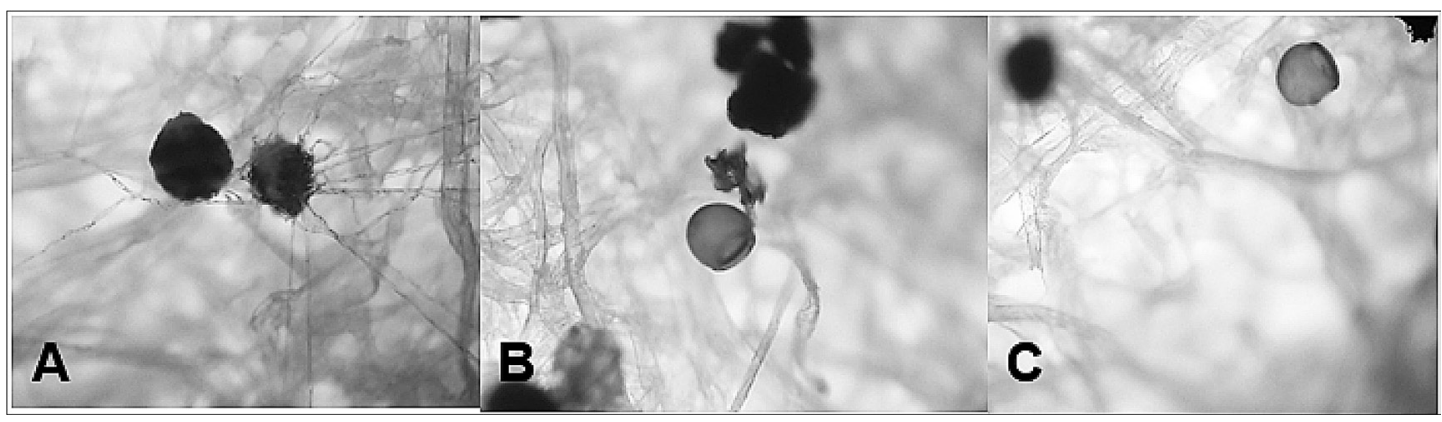

Figura 3. Morfología de las esporas observadas al microscopio. A) Espora negra con borde irregular de forma globosa circular y espora parda de forma globosa circular y con sus bordes lisos. B) Esporas amarillas con bordes lisos y forma globosa. C) Espora de color blanco con bordes regulares de forma circular (400X).

\section{Discusión}

La vainica y el frijol común pertenecen al grupo de las leguminosas, plantas caracterizadas por la fijación de nitrógeno de la atmosfera mediante la asociación con Rhizobium phaseoli, así como la fijación de fósforo gracias a la capacidad de asociarse con micorrizas, por lo que se utilizan como cultivo trampa para realizar ensayos con micorrizas (Gamboa, 2005). El efecto más importante que producen las micorrizas en los hospederos es un incremento en la absorción de nutrientes minerales del suelo, que se traduce en un mayor crecimiento y desarrollo. La principal causa de este efecto es la expansión del micelio externo del hongo por el suelo rizosférico, que permite la captación de los nutrientes más allá de la zona de agotamiento que se crea alrededor de las raíces (Paillacho, 2010).

En la investigación realizada por Aravena (2007) en plantas de aguacate y cítricos se observó que ninguna de las variables (altura, diámetro, materia seca radicular y aérea) afectó el crecimiento final de las plantas. Se conoce que las respuestas a la micorrización tienden a ser menores en suelos ricos en fósforo. La concentración de fósforo presente en el sustrato que se utilizó durante las pruebas no se midió, por lo que dicho elemento podría tener influencia en los resultados obtenidos en los ensayos.

Otro factor que se debe tener en consideración, y que eventualmente podría explicar la falta de diferenciación en el crecimiento de las plantas, es el tiempo transcurrido entre la inoculación y las mediciones realizadas. Plantas que se encuentran en condiciones de invernadero, con fertilización y riego controlados, y pese a porcentajes de colonización adecuados, pueden no expresar respuestas en el crecimiento debido al tiempo de exposición a la acción de las micorrizas, lo que genera un enmascaramiento de la efectividad de la micorrización (Aravena, 2007).

Dado que las micorrizas vesículo-arbusculares no se desarrollan en cultivos puros, se emplea una planta trampa susceptible a la micorrización, como la vainica, para la multiplicación de esporas del hongo (Paillacho, 2010). Para cultivos de crecimiento rápido como el maíz y el frijol, se deja el cultivo trampa por un periodo de tres (Paillacho, 2010) a cuatro meses (Habte \& Osorio, 2001). En las pruebas realizadas por Kohashi y Aguirre (2002), se obtuvo que 30 días después de la siembra las plantas de frijol común inoculadas empezaron a inducir un mayor desarrollo vegetal que el testigo. Para generar un cultivo trampa se debe utilizar una planta de crecimiento rápido, que se adapte a las condiciones de crecimiento, que sea colonizada por micorrizas fácilmente y que produzca una gran cantidad de raíces en un tiempo relativamente corto (45-60 días). Por estas y otras razones es que se seleccionó el frijol.

Para asegurarse de que la mayoría de las esporas en el inóculo estén maduras, es esencial el crecimiento del cultivo trampa durante 12 a 14 semanas (Habte \& Osorio, 2001). Tomando en 
cuenta los resultados obtenidos en cuanto al crecimiento, se hace necesario un estudio con un mayor tiempo de evaluación (más de un mes después de la siembra) para poder determinar mejor el efecto de la micorrización en el desarrollo de las plantas de vainica (Aravena, 2007). En cuanto al desarrollo radical, en un estudio en frijol se determinó que la edad de la planta afecta significativamente la masa radical fresca obtenida y que varía con el estado de desarrollo del cultivo, con lo cual se obtuvieron valores mayores en las plantas cosechadas a los 60 días que en plantas evaluadas a los 30 días, en donde los tratamientos no influenciaron la masa radical de las plantas de frijol (Galindo, 2008). La ausencia de diferencias significativas entre la longitud de las raíces en la investigación efectuada podría relacionarse con factores como el periodo de evaluación del ensayo, ya que se requiere un tiempo mayor para encontrar mejoras en el desarrollo radicular de las plantas de frijol.

A su vez, en el cultivo de trigo se determinó que la longitud radical y otras características morfológicas de una planta están establecidas genéticamente y también son influenciadas por las condiciones ambientales. De este modo, de acuerdo con la especie de planta, la longitud radical varía si se modifica la concentración de algún nutrimento del suelo, aunado a esto con el fin de evaluar eficiencia y obtención de nutrimentos del suelo, en las plantas de trigo evaluadas se obtuvo que la longitud radical fue un $23 \%$ mayor en un suelo natural que en un suelo estéril (Cruz et al., 1998). Estos resultados demuestran que la longitud radical se ve influenciada por la condición del suelo y la dosis de nutrientes presentes, especialmente fósforo, lo que podría ser una causa por la cual no se obtuvieron diferencias significativas en los tratamientos evaluados en relación con el crecimiento radicular en las plantas de frijol.

Phaseolus vulgaris se caracteriza por desarrollar micorrizas vesículo-arbusculares, por eso es común ver raíces colonizadas con arbúsculos y otras estructuras fúngicas (Guzmán et al., 1992). Esto justifica que se encontraran esporas y arbúsculos al teñir las raíces, tal y como se observa en la figura 2. Según Monroy (2004), la colonización se produce más rápidamente a partir de raíces previamente micorrizadas que a partir de esporas, ya que en la raíz tienen mayor viabilidad y desarrollo de estructuras. Sin embargo, en este ensayo ocurrió lo contrario, ya que el mayor porcentaje de colonización fue el tratamiento de inoculación de esporas, lo cual se puede justificar con lo obtenido estadísticamente, ya que el mayor número de esporas viables se obtuvo en los tratamientos de inóculo con raíces y esporas, ya que entre ambos no hubo diferencia significativa pero fueron las mayores medias. En un ensayo efectuado en maíz por Pérez et al. (2012), se obtuvo que a mayor número de esporas en el suelo, mayor porcentaje de colonización de hongos micorrízicos; esto coincide con los datos obtenidos, ya que el tratamiento que obtuvo mayor número de esporas fue el mismo que tuvo un mayor porcentaje de colonización.

Con respecto a la cantidad de esporas en los diferentes tratamientos, se observó que en todos hay presencia de ellas. La formación de esporas comienza entre la tercera y cuarta semana después de la colonización de la raíz. Sin embargo, la extensión de la esporulación se puede ver afectada por la planta hospedera, el suelo y las condiciones medioambientales (León, 2006). Los resultados muestran diferencias entre los tratamientos con respecto a la cantidad de esporas; los inóculos que favorecieron más la producción de estas estructuras reproductivas fueron los tratados con las esporas aisladas y los tratados con raíces, con un promedio de 233,6 esporas/20 $\mathrm{g}$ de suelo y 232,4 esporas/20 g de suelo, respectivamente, los cuales corresponderían a aproximadamente 1170 esporas/100 g. Con respecto a la cantidad de esporas aisladas, se ha reportado que puede variar de acuerdo con el tipo de uso del suelo; en el caso de esporas que se han recuperado de potreros, bosques o zonas de recuperación natural, el promedio es de aproximadamente 2000 esporas/100 g de suelo; mientras que en suelos que han sido cultivados (monocultivo o policultivo), los promedios del número de esporas disminuyen a alrededor de 1000 esporas/100 g de suelo. Esto es producto de la alteración de los ecosistemas naturales, ya que al darle diferentes usos y tratamientos al suelo, se influye en la abundancia de esporas y en la composición de especies de las micorrizas arbusculares (León, 2006; Moreira et al., 2012). 
A pesar de que los tratamientos no presentan diferencias significativas para la cantidad de esporas germinadas, las medias obtenidas en la prueba de Tuckey muestran que los tratamientos de inóculo con raíces y esporas presentaron la mayor cantidad de esporas germinadas, lo que evidencia la viabilidad de estas estructuras reproductivas de los hongos micorrízicos que colonizaron las raíces de las plantas de vainica.

Sin embargo, se debe tomar en cuenta que en el tratamiento testigo también se contabilizó una presencia importante de esporas, con un promedio de 121 esporas/20 g de suelo. Esto se puede deber a que en los procesos de esterilización de la tierra para cultivar las semillas, las esporas, por ser estructuras que presentan ciertos niveles de resistencia, no se destruyen por completo, en especial las esporas sexuales que usualmente presentan mayor resistencia al calor que las asexuales (García, 2004).

Al estudiar la abundancia y diversidad de micorrizas presentes en una planta hospedera mediante el aislamiento de las esporas, se observa que existen varios factores que pueden afectar la caracterización. Es por eso que se deberían tomar en cuenta aspectos como la capacidad de cada especie para producir esporas, la época y las condiciones del muestreo (León, 2006). A partir de la morfología particular de las esporas observadas se puede determinar que en la rizosfera hay presencia de varias especies de micorrizas, lo que demuestra la diversidad de estos hongos en los suelos de cultivos de frijol.

La morfología de las esporas se puede caracterizar de acuerdo a la coloración, el tamaño y la forma, como se observó en la figura 3. En este caso, se realizó una clasificación básica a partir de la mayor diferenciación observable, que es el color de la espora, y según esta característica se observaron cuatro grupos diferenciados: 1. Esporas negras con borde irregular de forma globosa circular, 2. Esporas pardas de forma globosa circular y con sus bordes lisos, 3. Esporas amarillas con bordes lisos y forma globosa y 4. Esporas de color blanco con bordes regulares de forma circular. Esta agrupación a partir del color no corresponde a ningún tipo de clasificación taxonómica, ya que es necesario contar con una mayor y detallada cantidad de datos para este fin.

\section{Conclusiones}

La longitud del tallo y de la raíz de las plantas evaluadas no presentó diferencias estadísticamente significativas entre los distintos tratamientos.

El método de inoculación con esporas presentó el mayor porcentaje de micorrización y colonización, en comparación con la muestra inicial utilizada para el aislamiento de las esporas.

Los métodos de inoculación que favorecieron más la producción de estructuras reproductivas fueron los tratados con las esporas aisladas y los tratados con raíces; de igual forma, ambos presentaron la mayor cantidad de esporas germinadas.

Para la elaboración de algún producto comercial a base de micorrizas, se recomienda utilizar como inóculo las raíces de plantas infectadas, ya que, a pesar de que el inóculo con esporas aisladas también presentó resultados satisfactorios, el proceso metodológico para el aislamiento es complicado e implicaría mayor inversión de trabajo, tiempo y dinero. Además, para futuros estudios se recomienda no utilizar tierra como sustrato para los cultivos trampa, sino algún otro tipo de material, como arena, que es menos probable que tenga esporas que vayan a influenciar los resultados finales.

\section{Bibliografía}

Aravena, C. (2007). Efecto de la micorrización en plantas de vivero de palto y cítricos bajo diferentes dosis de fertilización. Obtenido de http://www.avocadosource.com/papers/Chile_Papers_A-Z/A-B-C/AravenaCristian0000.pdf 
Cuadros, G.A., Gómez, R. \& Rodríguez, N.F. (2011). Asociación simbiótica entre hongos micorrízicos arbusculares y el sistema radicular de plántulas de cacao (Theobroma cacao L.): efecto de la formononetina y la disponibilidad de fósforo en el suelo. Corpoica Ciencia y Tecnología Agropecuaria, 12(1), 77-85. Obtenido de http:// www.corpoica.org.co/sitioweb/Archivos/Revista/8_Asociacin.pdf

Cruz, I., Gayosso, E., Cruz, G., Ortiz, I. \& Manske, G. (1998). Colonización micorrízica arbuscular, actividad fosfatásica y longitud radical como respuesta a estrés de fósforo en trigo y triticale cultivados en un andisol. Terra, 16(01), 55-61. Obtenido de http://www.redalyc.org/articulo.oa?id=57316107

Cruz, Y., García, M., León, Y. \& Acosta, Y. (2014). Influencia de la aplicación de micorrizas arbusculares y la reducción del fertilizante mineral en plántulas de tabaco. Cultivos Tropicales, 35(01), 21-24. Obtenido de: http://scielo. sld.cu/pdf/ctr/v35n1/ctr03114.pdf

Galindo, P. (2008). Comparación del efecto de inoculación con micorrizas vesículo-arbusculares nativas y comerciales en plantas de frijol (Vigna unguiculata (L.) WALP). Tesis de Grado. Universidad de Zulia, Venezuela.

Gamboa, W. (2005). Producción agroecológica: una opción para el desarrollo del cultivo del chayote. San José: Editorial de la Universidad de Costa Rica.

García, V. (2004). Introducción a la microbiología. San José: Editorial Universidad Estatal a Distancia.

Guzmán, R., Bethlenfalvay, G. \& Ferrera, R. (1992). The role of V-A mycorrhizae in the transfer of root exudates between associated bean and corn plants under field conditions. Obtenido de http://agris.fao.org/agrissearch/search.do?recordID=MX19940123769

Habte, M. \& Osorio, N.W. (2001). Arbuscular mycorrhizas: producing and applying arbuscular mycorrhizal inoculum. Obtenido de http://www.ctahr.hawaii.edu/oc/freepubs/pdf/amf_manual.pdf

Kohashi, J. \& Aguirre, J. (junio, 2002). Dinámica de la colonización micorrízica y su efecto sobre los componentes del rendimiento y contenido de fósforo en frijol común. Agricultura Técnica en México, 28(1), 23-33. Obtenido de http://www.redalyc.org/pdf/608/60828103.pdf

León, D. (2006). Evaluación y caracterización de micorrizas arbusculares asociadas a yuca (Manihot esculenta sp.) en dos regiones de la Amazonía colombiana. Tesis para optar por el título de microbióloga agrícola y veterinaria. Pontificia Universidad Javeriana.

Liriano, R., Núñez, D. \& Barceló, R. (octubre, 2012). Efecto de la aplicación de Rhizobium y Micorriza en el crecimiento de frijol (Phaseolus vulgaris L) variedad CC-25-9 negro. Centro Agrícola, 39(4), 17-20. Obtenido de http://cagricola.uclv.edu.cu/descargas/pdf/V39-Numero_4/cag044121877.pdf

Mohammadi, K., Khalesro, S., Sohrabi, Y. \& Heidari, G. (2011). A Review: Beneficial effects of the mycorrhizal fungi for plant growth. Journal of Applied Environmental and Biological Sciences, 1(9), 310-319. Obtenido de http:// www.textroad.com/pdf/JAEBS/J.\%20Appl.\%20Environ.\%20Biol.\%20Sci.,\%201(9)310-319,\%202011.pdf

Monroy, H. (2004). Caracterización de hongos formadores de micorrizas arbusculares (HMA) nativas, en seis coberturas de cítricos en el Piedemonte del Meta. Tesis de Grado. Universidad Nacional de Colombia.

Moreira, F., Huising \& Bignell. (2012). Manual de biología de suelos tropicales. Muestreo y caracterización de la biodiversidad bajo suelo. México, D.F.: Instituto Nacional de Ecología.

Paillacho, F.I. (2010). Evaluación de la efectividad de las micorrizas arbusculares nativas sobre el desarrollo y estado nutritivo del palmito en etapa de vivero, en Santo Domingo de los Tsáchilas. Tesis de maestría. Escuela Politécnica del Ejército, Santo Domingo de los Tsáchilas, Ecuador. Obtenido de http://repositorio.espe.edu.ec/ bitstream/21000/2892/1/T-ESPE-IASA\%20II-002332.pdf

Pérez, A., Rojas, J. \& Montes, D. (septiembre, 2011). Hongos formadores de micorrizas arbusculares: una alternativa biológica para la sosteniblidad de los agroecosistemas de praderas en el Caribe colombiano. Revista Colombiana de Ciencia Animal, 3(2), 366-385. Obtenido de http://www.recia.edu.co/documentos-recia/vol3num2/revisiones/REC-03-02-REV-2-MA-HONGOS.pdf

Pérez, Y., Álvarez, J., Mendoza, J., Pat, J.M., Gómez, R. \& Cuevas, L. (2012). Diversidad de hongos micorrízicos arbusculares en maíz con cultivo de cobertura y biofertilizantes en Chiapas, México. Gayana, Botánica 69(1), 46-56.

Terry, E., Ruiz, J., Tejeda, T. \& Díaz, M. (2013). Respuesta del cultivo de la habichuela (Phaseolus vulgaris L. var. Verlili) a la aplicación de diferentes bioproductos. Cultivos Tropicales, 34(3), 5-10. Obtenido de http://scielo. sld.cu/pdf/ctr/v34n3/ctr01313.pdf

Ulloa, J., Rosas, P., Ramírez, J. \& Ulloa, B. (julio, 2011). El frijol (Phaseolus vulgaris): su importancia nutricional y como fuente de fitoquímicos. Revista Fuente, 3(8), 5-9. Obtenido de http://fuente.uan.edu.mx/publicaciones/03-08/1.pdf 